\title{
Ang Pagsasalin Bilang Malikhaing Pagsulat
}

U Z Eliserio

\author{
Unibersidad ng Pilipinas, Diliman
}

\section{Abstract}

This paper argues for a view of translation as creative writing, doing so via a reading of three texts clustered around the signifier "Nietzsche". The first part of the paper is an examination of the philosophical translations Friedrich Nietzsche deploys in his analyses of ethics, power, and nations. Slaves, according to Nietzsche, changed the meaning of the word "good," shifting its referent from the "masters," those who were strong, wealthy, cruel, to the "slaves," who were weak, slow, and helpless. This is the slave revolt in morals that, for example, changed the meaning of weakness. The weak man, the slave, is no longer unable to act, but "chooses" not to act, our of humility and forgiveness. The second part of the paper presents the literary appropriation of Nietzsche by Faustino Aguilar in his novel Nagalunod sa Katihan. Translating mainly from Nietzsche's Thus Spoke Zarathustra, Aguilar distorts and decontextualizes Zarathustra's aphorisms and sayings, going so far as to turn a derogatory term into praise. Nietzsche philosophy for individuals roaming the intellectual mountains of Western Europe is deconstructed, given new life, an afterlife, as one of many tools in Aguilar's project for envisioning a modern Filipino nation. The third part of the paper is a meditation on the nature and 
aims of translation. To be faithful to Nietzsche's views on power, one must not be stuck to the letter of his texts. Translation is an act of power, and there are slavish and masterful approaches to the act. Translation, in this paper, is not treated as dry process, but instead as an opportunity for creation. It is not the repetition of a text from one language to another, but the bringing forth of something new.

\section{Keywords}

Nietzsche, translation, Faustino Aguilar, Nagalunod sa Katihan, slave morality 


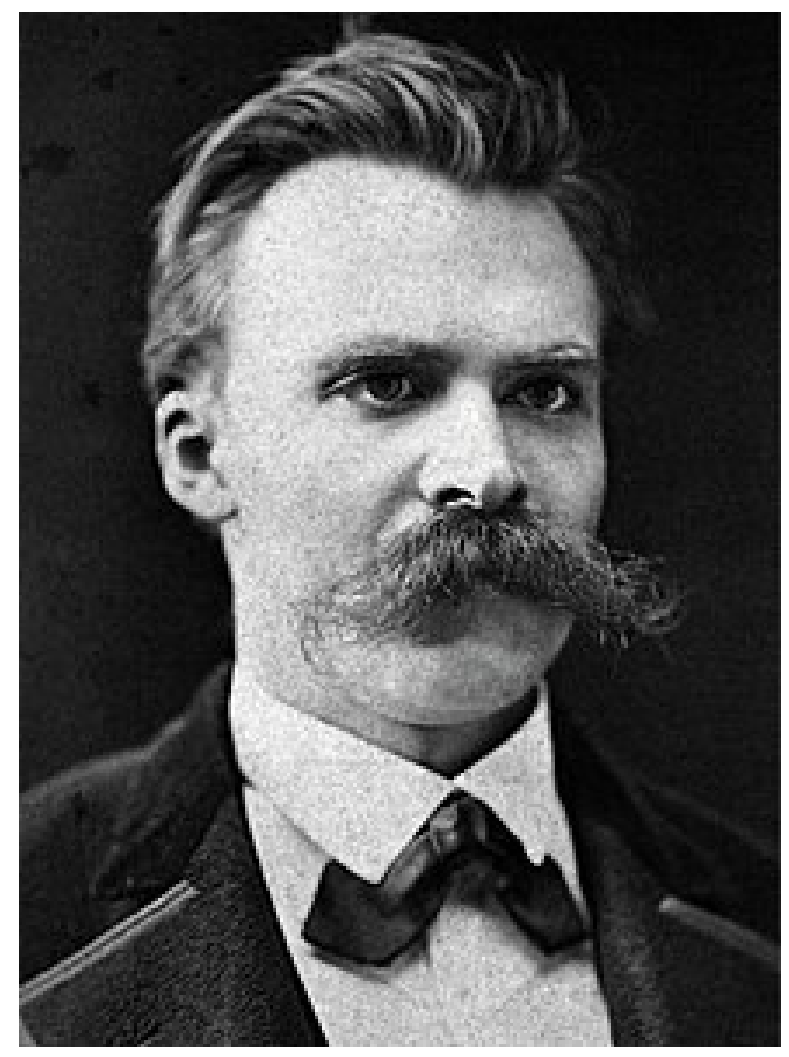

Friedrich Nietzsche 
Ang papel na ito ay maka-Nietzscheng dulog sa pagsasalin bilang anyo ng malikhaing pagsulat. Binubuo ito ng tatlong bahagi: una, ilalarawan ang mga pilosopikal na pagsasalin ni Friedrich Nietzsche. Sa kanyang mga sulatin, madalas ipinapakita ni Nietzsche ang isang konsepto mula sa punto de bistang iba sa nakasanayan, para ilantad ang mga nakatagong prehuwisyo nito laban sa itinuturing niyang malakas at malusog na uri ng buhay, at ang pagkiling nito sa mahina at pagod na uri ng buhay. Nananatili ang titik ng konsepto, pero iba na ang espiritu nito. Nabaklas na ni Nietzsche. Pangalawa, ilalarawan ang iba't ibang isyu ng pagsasalin na umiikot sa pangalan ni Nietzsche. Kasama sa diskusyon sa malayang salin ni Faustino Aguilar ng ilang aporismo mula sa Ito ang Salita ni Zarathustra, ang mga komentaryo ng pinakatanyag na tagasalin ni Nietzsche sa Estados Unidos, si Walter Kaufmann, tungkol sa pagkakamali ng mga naunang nagsalin kay Nietzsche, at gayundin ang relasyon ng pagsasalin sa interpretasyon. Sa huling bahagi, palalawigin at pasasabugin ang konsepto ng "reaktibo" sa Tungo sa Genealogy ng Moralidad ni Nietzsche para maglatag ng pilosopikal na pagtatanggol sa pagsasalin bilang malikhaing pagsulat. Bibigyang-diin dito ang pangangailangan sa pagiging bukas at eksperimental sa pagsasalin.

\section{Pag Nagsalin si Nietzsche}

Ang buong Genealogy ay maaaring tawaging ehersisyo sa pagsasalin. Dobleng salin pa nga. Unang pagsasalin ang pagtransporma ng mga alipin ng "mabuti” ng mga amo, tungong "buktot." Pangalawang pagsasalin ang ginawa ni Nietzsche. Isinalin niya ang "Moralidad," malaking titik "M", tungong pang-aliping "moralidad." Ibang uri ng halagahan ang nasa likod ng ebalwasyong "mabuti at masama" at "mabuti at buktot." Ang huli ay mga kategorya ng pang-aliping moralidad, na reaktibo. Masasaktan muna ang alipin, at tatawagin niyang "buktot" ang nanakit sa kanya. Saka pa lamang maluluwal ang ebalwasyong "mabuti," na iaaplay sa sinaktang alipin. Iba ang "mabuti" ng alipin sa "mabuti" ng amo. Nauunang tawagin ng amo na "mabuti" ang kanyang sarili, ang hindi katulad niya ang "masama" (Nietzsche 460-492). Ang sasabihin ng amo, mabuti ang malakas kasi malakas ang amo. Pero ang alipin, nasaktan nga ng amo, tatawaging buktot ang malakas, at 
siyang kabaliktaran, siyang mahina ang mabuti. Nakikita natin ang sayaw na ito sa ebalwasyon ng pagkamakasarili at pagiging mapagkumbaba. Sa mga ganitong halagahan nakabase kung sa anong uri ng buhay tayo ooo at hihindi. Paliwanag nga ni Deleuze sa edisyong Ingles ng kanyang Nietzsche and Philosophy: "For any proposition is itself a set of symptoms expressing a way of being or a mode of existence of the speaker, that is to say the state of forces that he maintains or tries to maintain with himself [sic] and others.... In this sense a proposition always reflects a mode of existence, a "type." What is the mode of existence of the person who utters any given proposition, what mode of existence is needed in order to be able to utter it?” (x).

Maaaring sabihing malaking aspekto sa obra ni Nietzsche ang pagsasalin. Sinabi niya, halimbawa, sa Ang Kapanganakan ng Trahedya na bilang penomenong estetik lamang maaaring maging lehitimo ang buhay at ang mundo (52, 141). Binabago rito ang kahulugan ng tao, ng pag-iral. Imbes na, kunwari, regalo ng Diyos, ang buhay ang likhang-sining na. Pag naging ganito ang punto de bista, maaari nang bigyan ng saysay ang paghihirap. Oo, malungkot na nagkakanser ang iyong anak. Pero, wow-ang tindi ng emosyon! Suntok sa tyan ang development na ito sa banghay ng iyong kwento. Pagsasalin ba ito o interpretasyon? Ano nga ba ang pagkakaiba ng dalawa?

Tingnan natin ang isa pang pagtatangka ni Nietzsche na magsalin. Ngayon naman sa Ito ang Salita ni Zarathustra. Sa ikatlong aklat, sa seksyong pinamagatang "Hinggil sa Tatlong Kabuktutan," itinanong ni Nietzsche/ Zarathustra kung ano ang tatlong bagay na pinakaisinusumpa sa mundo. Ang sagot, sabi niya, ay pagtatalik, pagnanasang mamuno, at pagkamakasarili. Tinimbang niya ang tatlong bagay na ito, at bininyagan silang banal. Kasi nga, ang pagtatalik, halimbawa, "para sa mga tuyot, matamis na lason; para sa may kalooban ng leon, pampasigla ng puso" (Nietzsche 300). Tinuligsa naman ng mga pekeng-madunong ang pagkamakasarili (303).

Ano nga ba ang pagkakaiba ng pagsasalin at interpretasyon? Sa unang sulyap, para bang magkaiba sila. Ang pagsasalin, di-umano, ay pagpili kung aling salita sa wikang A ang katumbas ng salitang B sa wikang C. Kaya, halimbawa, ang "pusa” ay "gato" (o "gata”!). Samantala, ang interpretasyon naman, ang itinatanong, imbes na “Ano ang katumbas nito?”, “Ano ang 
kahulugan nito?” Halimbawa, “Ano ang kahulugan ng Tungo sa Genealogy ng Moralidad?" Pwede itong sagutin ng "Ang pagiging mapagkumbaba ay pang-aliping birtud!” O di kaya, "Dapat pangmalakas ang ating halagahan.” Pero, alinsunod nga sa Genealogy ni Nietzsche, maaari nating itanong, anong uri ng buhay ang itinutulak ng paghihiwalay sa mga konseptong pagsasalin at interpretasyon, at anong uri ng buhay naman ang maaari tayong magkaroon kung pag-iisahin natin ang dalawang ito? Ano pa ang pwede nating makita lampas sa unang sulyap?

Kunin natin ang "pusa." "Gato” ba ito o "gata”? Kung jenerik na pusa, sasabihin na "gato" dapat ang salin. Pero bakit lalakeng pusa ang itinuturing na jenerik na pusa? Bakit "he" ang salin kung hindi espisipikong babae ang "siya”? Ang "tao" ba ay "man," "woman," o "human"? Paumanhin sa mistulang pagbabalik-aral sa feministang pananaw sa wika. Paalala lang ito na hindi simpleng paghahanap ng mga magkakatumbas na salita ang pagsasalin.

Mahirap minsan ibenta ang interpretasyon. Madalas igigiit ng madla na ang isang bagay ay kung ano lang ito talaga. Ang gusali ay gusali lamang, hindi ito tite. Pinalalambot ng pagsasalin ang kasiguraduhang ito. Kasi nga, sa pagsasalin, ang "bitch" ay hindi lang "bitch,” kundi "puta.” Pag pinaghugpong ang interpretasyon at pagsasalin, ang "bitch" ay pwede nang "mahusay." Kasi nga, "bitch" = "bad ass" = "mahusay." Ang "that's a bitchin' car" ay "iyan ay napakagandang kotse," halimbawa. Pag nagsasalin ka, hindi maiiwasang mag-interpreta. At ang interpretasyon ay isang uri ng pagsasalin (intrawika).

Tinututulan ni Nietzsche ang pagsasaling ginagawa ng mga tinatawag niyang sikolohistang taga-Inglatera, nang pagtumbasin nila ang "mabuti" at "may gamit" (460-463). Tinututulan niya rito ang pilosopiya ng yutilitaryanisim, na itinutumbas ang "mabuti” sa "masarap" (nagagamit tungo sa sarap). Mabuti ang pagtutulungan, halimbawa, kasi lahat yumayaman pag nagtulungan. Hindi rito mahalaga kung totoo o hindi itong sinabi ko. Ang mahalaga para kay Nietzsche, anong uri ng buhay ang ibinibida nito?

Ano nga bang uri ng tagasalin ang ibinibida pag igigiit na tumbasan ang pagsasalin? Iyong gusto ay strikto, iyong may standard. Pwede nating sabihin, tagasalin itong hindi flexibol. Kung mas positibo ang ating pagbebenta, pwede nating sabihin, tagasalin itong tapat sa teksto, tapat sa orihinal. 
May silbi naman ang ganitong uri ng tagasalin. Pag nagda-drive ka sa Madrid at sinabi sa iyo ng Espanyol na "Gira a la derecha" at isinalin ito ng app mo bilang "Dumiretso ka” imbes na "Kumanan ka," baka bumangga ka sa pader.

Pero hindi naman kasi tumbas ang pagda-drive sa moralidad, at pilosopiya. Syempre pa, maaari nating kwestyunin ang halagahan sa likod ng kalituhan sa pagitan ng mga ordinaryong bagay (tulad ng pagbibigay ng direksyon) at mas importanteng bagay (tulad ng pagbibigay ng direksyon sa buhay). Pero wag na 'yon. Sa ibang araw na lang ang tanong na 'yon. Itanong na lang muna natin ngayon, ano ang mangyayari pag isinalin sa Filipino si Nietzsche? Ano ang pinakamalalang pwedeng mangyari?

\section{Pag Isinalin si Nietzsche}

Sa pagsasalin kay Nietzsche, kailangang itanong, bakit ginagawa ang pagsasalin na ito? Siyang nagsabing kailangang itanong kung ano ang halaga ng mga nakasanayan nang halagahan, kailangang tanungin, "Ano ang halaga mo?" Sa lahat naman ng pwedeng isalin na hirit sa Ito ang Salita ni Zarathustra, bakit pinili ni Faustino Aguilar na isalin ito?: "Sinabi ng isang pantas: may kasama kang babai? Huwag mong kalilimutan ang pamalo” (34). Hindi ito payo ni Nietzsche/Zarathustra, kundi ng isa pang tauhan sa Zarathustra, isang matanda (babae pa nga!). Baka naman naglalaro lang si Aguilar. Nakakatawa nga naman ang linya. Pero paano naman itong isa pa niyang isinalin mula kay Nietzsche?: "Dati-dati ang kalakasan ay tinawag na Diyos, bago tinawag na tao, ngunit ngayo'y ang bayan na” (111). Salin ito mula sa unang aklat ng Zarathustra, pinamagatang "Hinggil sa Pagbabasa at Pagsusulat." Narito ang Ingles: "Once the spirit was God, then he became man, and now he even becomes rabble" (Nietzsche 152). Sa espiritu ng pagkontra sa demokrasya ang inusal ni Nietzsche/Zarathustra ang pangungusap. Mahihinuha ito sa konteksto ng sipi: "Another century of readers-and the spirit itself will stink. That everyone may learn to read, in the long run corrupts not only writing but also thinking." Hindi lang binaliktad ni Aguilar ang kahulugan ng isinulat ni Nietzsche; iniwan na niya ang kahulugan nito. O, sa madaling sabi, nag-interpreta siya kung kailan ang inasahan lang natin niyang gagawin ay magsalin. Kaya nga naging "bayan” ang "rabble.” Hindi mahalaga kung 
ano ito sa orihinal na Aleman ("Pöbel" at hindi "Volk" para sa makulit). Ang mahalaga ay ang hindi pagpapahala ni Aguilar sa intensyon ni Nietzsche, o ang kahulugan ng orihinal. Kasi meron siyang ibang layunin, at iyon ay ang paglalatag ng kanyang diskurso tungkol sa bayan: "Ang bayan ay hindi ang pook na sinilangan lamang ng isang tao, ang bayan ay iyong bahagi ng lupang bukod sa kinakitaan ng unang liwanag ay nag-iwi pa sa unang pag-ibig, sa unang pangarap, sa unang tibok ng marangal na damdamin ng lahat na mga kalahi. Ang pag-ibig sa bayan ay hindi sa salita lamang dapat ipakita kung hindi sa gawa” (Aguilar 103). May panganib ba sa ganitong uri ng pagsasalin? Kung ang ang "rabble" ay maaaring maging "bayan," anong pipigil sa kung sinong freshman sa pagsabing ang "slavery" ay "kalayaan"? At kung sa interpretasyon, ang isang tore, kahit walang ebidensyang kontekstwal, walang basehan sa intensyon ng awtor, ay sasabihin nating tite, saan pa tayo titigil? Hindi ito anarkiya kundi gulo. Para saan pa ang pakikipag-usap, ang pagsusulat at pagbabasa? Meron pa bang komunikasyon?

Syempre pa, ang malayang salin, ang salin-bilang-interpretasyon, hindi nagaganap sa kung saang metapisikal na baitang. Wala nga namang pipigil sa isang freshman sa pagsabing ang "slavery" ay "kalayaan," at ang tore ay tite. Babagsak nga lang siya sa kanyang klase. Hindi naman salawikain ang mga isinalin ni Aguilar, bahagi ito ng kanyang nobela. Salungat sa sintido komun ang ginagawang malayang salin dahil may ninanais na ikomyunikeyt. Hindi ito katapusan kundi simula ng pagsusulat at pagbabasa. Si Nietzsche/ Zarathustra na mismo ang nagsabi, "every people speaks its tongue of good and evil, which the neighbor does not understand. It has invented its own language of customs and rights" (161). Sa espiritu ni Aguilar, out of context ang pagsiping ito. Sa espiritu rin ni Aguilar, ipapaliwanag ko ang lakas ng malayang salin.

Ang binubuo rito ay isang diskurso. Ang mga teksto ay pinaglalaruan, iniinterpreta, isinasalin nang may ibang layunin bukod sa nakasanayan: panloob na kritisismo sa orihinal, parodiya, travesti, pagsasanay sa isang wika na sumunod sa batas ng isa pang wika, pang-uusig, pagpapalawig ng bokabularyo ng Filipino, pagturo sa pilosopiya kung paano mag-Filipino. Inaasahan na gagawin rin sa salin ko ang ginawa ko sa likha ni Nietzsche. 
Pero inaasahan ding may mga striktong mambabasa, ang mga tinatawag ni Nietzsche na "philosophical laborers," na magsusubok magtakda ng standard, ng orthodox na pagbabasa dito sa aking mga isinusulat. Samantalang ang tumbasang salin, sinusupil ang potensyal sa paglalaro, hinahalina ng malayang pagsasalin ang seryosong pagbabasa.

Tutal naman ay naamin ko nang hindi ako marunong mag-Aleman, tumungo tayo sa isang wikang inaasahan kong hindi kayang salitain ng aking mga mambabasa: Griyego. Griyego sa Bibliya. Naengkwentro ko ang kasong ito sa talahuli ng librong Redeeming Nietzsche ni Giles Fraser. Pari ng Iglesia ng Inglatera si Fraser, at ipinaliwanag niya na ang " $\alpha \nu \omega \theta \varepsilon \nu$ ” ay nangangahulugang "ulit" at "mula sa itaas." Binigyang-diin niya ang retorikal na pagbabasa sa Bibliya. "The author of the Gospel is clearly making fun of Nicodemus' stupidity [kasi nga akala ni Nicodemus "ulit" ang tinutukoy ni Hesus sa pagsabing “ $\alpha \nu \omega \theta \varepsilon v$," pero sa totoo ay "mula sa itaas” ang ibig sabihin ng Panginoon]. ... Ironic then that so many 'Bible-believing' Christians believe that being "born again" is a precondition of being saved" (175).

Sa isang banda, namimintis ni Fraser ang mala-Nicodemus na katangahan ng mga Kristyanong Born Again. Oo, "maling salin” nga ito, pero may retorikal pa rin itong kapangyarihan. Pag nga naman naging Kristyano ka, para kang ipinanganak muli, kasi magbabago ang buhay mo. Gayundin, mintis ang kritisismo ni Fraser kay Nietzsche. Sinabi niya kasi na naïve ang pilosopiya ni Nietzsche, dahil wala itong lugar para sa "tae." Ang taeng tinutukoy ni Fraser dito ay paghihirap, karahasan, gera. Ang pag-iisip kasi ni Nietzsche ay naganap bago ang mga kalagim-lagim na kaganapan ng ika-20 siglo, halimbawa, ang Holocaust. Pang-inosenteng panahon daw ang mga ibinibidang ideya ni Nietzsche, ayon kay Fraser. Pero hindi naman natin masisisi si Nietzsche na nabuhay at nagsulat siya sa panahon bago mag-Holocaust. Ang sinubukan kasing gawin ni Fraser ay bigyan ng striktong interpretasyon si Nietzsche, iyong may pagtukoy sa kanyang mga intensyon (intensyon ni Nietzsche, hindi ni Fraser). Siguro huli na nang matuklasan ni Fraser na lupaypay pala ang pilosopiya ni Nietzsche sa harap ng tae ng ika-20 siglo (nabasa na niya siguro ang obras completas ni Nietzsche). Kaya inilatag niya ang striktong interpretasyon, tinimbang ito, 
at idineklarang kulang. Pero bakit pa? Ang mas produktibo sigurong dapat niyang ginawa, inakma si Nietzsche sa ating panahon. Sumulat dapat siya ng Nietzsche para sa Panahon pagkatapos ng Holocaust. O, Nietzsche: Pangtaeng Pananaw. Kinailangan niyang irepresenta ang striktong interpretasyon niya kay Nietzsche para magsilbi itong foil sa kanyang binibidang diskurso ng kanyang bersyon ng Kristyanismo, na may pakikiramay at malasakit sa sangkatauhan. Pagtakas kasi ang ginagawa ni Nietzsche, kahit gaano kamacho niya ifreym ang pagtakas na ito. "Nietzsche's rejection of pity [...] represents a rejection of, a refusal to acknowledge, any sense of identity between himself and ... suffering humanity. Better to murder the dwarf, to oust him, to sacrifice him even, than to have him make me face my own dwarfness-thus spoke Zarathustra. In rejecting suffering humanity, in casting people as the herd, Nietzsche is seeking to set himself free from the earth below" (Fraser 2002, 163). Para tuloy mapanlinlang na mambabasa ni Nietzsche si Fraser. Pero may isa pang paraan ng pagbabasa sa kanyang ginawa. Kinailangang itumba ni Fraser ang figura ni Nietzsche na kanyang ginawa, ang FraserNietzsche, nang sa gayon ay makapagtayo ng ibang figura, Fraser-Hesus o Fraser-Kristo, na magagawa ngang harapin ang paghihirap na tinalikuran ni Nietzsche. Ang Nietzsche para sa panahon pagkatapos ng Holocaust ay itong Fraser-Hesus o Fraser-Kristo na ito. Si Fraser-Nietzsche ay kailangang mamatay para muling bumangon si Fraser-Kristo. At iligtas tayong lahat?

Sa ortodox na pagsasalin, tinatangkang palitan ang signifier habang pinapanatili ang signified. Halimbawa, ang "aso" at "perro" ay magkaibang signifier, pero ang signified nila pareho ay iyong hayop na may apat na paa, may buntot, malapit sa tao, galit sa pusa. Sa himagsikang pang-alipin sa moralidad, kung pwede nating isalin si Nietzsche, sinusubukang panatilihin ang signifier, habang pinapalitan ang signified. Samantalang ang "mabuti" ay tumutukoy sa mga amo, sa pang-among moralidad, sa pang-aliping moralidad, tumutukoy na ito sa mga alipinin. Sa pang-among moralidad, ang mabuti ay iyong malakas, at mabangis. Sa pang-aliping moralidad, ang mabuti ay iyong mapagkumbaba. Binigyan ito ng maka-kanang interpretasyon nina Robert Solomon at Kathleen Higgins sa kanilang What Nietzsche Really Said: "They see themselves as oppressed. They see themselves, in modern terms, as 
victims. Nor do they see the masters as merely happy and fulfilled. The slaves see them as oppressors, as people with the wrong values, the wrong ideals, the wrong ideas about living" (111). Pero kung ituturing pa rin nating pagsasalin ang ginawa ni Aguilar, anong uri ng interpretasyon na ngayon ang pwede nating gawin?

Awa sa tao ang huling umakit kay Zarathustra. Naigpawan ba ni Nietzsche ang pang-aakit? Inatake niya ng paulit-ulit ang awa sa kanyang mga isinulat. Pero bakit nga ba siya nagsulat? Pinupuri niya ang lakas, ang indibidwalismo. Kailangan nga raw harapin ang walang hanggang pagbabalik, ang walang hanggang pag-uulit. Sasabihin nga raw natin sa nakaraan, hindi lang "Iyon ang nangyari," kundi, "Iyon ang aking ninais." Kung gayon, bakit siya galit na galit na nagwagi ang pang-aliping moralidad? Bagaman hindi naman niya sinasabing dapat bumalik tayo sa pang-among moralidad, nakukulangan pa rin siya sa pang-aliping moralidad, kaya nga niya ito inuusig. Para saan? Bakit hindi niya masabi sa himagsik pang-alipin sa moralidad na "Iyon ang aking ninais"? Hindi man lang nga niya masabing "Iyon ang nangyari." Ang nasasabi niya, "Iyon ang karumal-dumal na nangyari." Bakit hindi na lang niya sinarili ang kanyang natuklasan, kung ganoon lang ang kanyang papuri sa pagiging makasarili? Shering is kering, kung gayon ba't siya nagsubok magkomyunikeyt sa kanyang mga mambabasa? Nangingisda ba siya para sa mga katulad niya? Katulad niyang ano?

Makapangyarihan ang paraan ng pagsusulat ni Nietzsche, pero suspetsa ko, pagkatapos masarapan sa pagbabasa, hindi na malaman ng mga iskolar kung ano ang gagawin sa kanya. Magkokomentaryo? Pero iyon nga ay para sa mga "philosophical laborers," hindi talaga mga pilosopo kundi mga manggagawa. Mahirap namang isabuhay ang mga konsepto tulad ng "lipat-tao" at "pang-among moralidad." Mas madali siguro ang pagsubok na maging maka-Nietzsche sa larang ng pag-iisip, ang "pamimilosopiya gamit ang martilyo.” Ito nga ang naging dilema ng mga akademikong kasama sa antolohiyang Nietzsche, Genealogy, Morality. Ayon sa editor, kalahati ay pumaksa sa "genealogy." Ang kalahati naman ay pumaksa sa moralidad. Sa 13 na sanaysay, dalawa ang may pagsalungat kay Nietzsche, samantalang 11 ang nagsubok palawigin ang mga konseptong inilatag sa Genealogy (Schacht 
xii-xiii). Ito ngayon ang interpretasyon. Kung hindi ka sasalungat, kailangan mong magdagdag. Kung hindi, bakit ka pa magsusulat? Kung wala ring bago tungkol kay Nietzsche na mahahagilap sa iyong komentaryo sa kanya, bakit hindi na lang si Nietzsche ang basahin (at muling basahin) ng mambabasa?

Sa kanyang pagsalin ng "rabble" bilang "bayan," hindi lang binago ni Aguilar ang signifier at pinanatili ang signifier, binago niya pati ang signified! Alinsunod dito, sa malayang salin, sa mapagpalayang salin, hindi lang babaguhin ang signified, babaguhin pati ang signifier!

Ang pagsasaling strikto, na minsan ay tinatawag na pagsasaling tapat, ay "teolohikal." Pwede pa nga nating sabihing pang-alipin. Kailangang sundin ang "amo" (ang orihinal na teksto). Teolohikal ko itong tinatawag dahil ang pagtatangkang panatilihin ang orihinal ay obsesyon ng mga pari at relihiyoso. Ang Bibliya kasi ay salita ng Diyos, at ang pagbabasa ng Bibliya ay pagtatangkang matuklasan kung ano ang nais ng Diyos mula sa atin. Kailangang maging eksakto sa pagsasalin dahil baka may masingit ang tao (ang nagsalin) sa salita ng Diyos. Ito ay karikatura lamang. Marami nang nasabi si Eugene Nida tungkol sa pagsasalin ng mga idyoma ng Bibliya at hindi na iyon kailangan pang ulitin dito (pero tingnan ang Nida 2000, 135, 137-140). Imbes, basahin na lang natin ang tindig ng isang Heswita, si Rene Javellana, na sumusunod sa panawagan ni Nida para sa "dynamic equivalence.” Paliwanag ni Javellana kay Nida, "kailangang hanapin ang katumbas sa kasalukuyang kultura ng isang bagay na natatagpuan sa kulturang Biblikal. Halimbawa, sa MBB ["Magandang Balita Bibliya”], isinasalin ang "hardness of heart” ng wikang Griyego na "katigasan ng ulo," sapagkat sa antropolihiya ng Bibliya ng "puso" ay inilalarawan na sentro ng pagpapasiya sa halip na sentro ng damdamin o emosyon" (158).

Kahit ang mga relihiyoso, pwede nating sabihin, ay hindi nagpapaalipin sa kanilang isinasaling teksto, bagkus ay ginagamit na kompas ang kanilang layunin. Isinalin ang "puso" bilang "ulo" para lalo pang mapalapit ang Filipinong mambabasa sa Diyos. Isinalin naman ni Aguilar ang "rabble" bilang "bayan" dahil nais niyang bumuo ng diskurso tungkol sa bayan.

Sa anong paraan makakapagdagdag sa pilosopiya at etika ang ganitong uri ng pagtingin sa pagsasalin? Para masagot ito, sagutin muna natin ang 
tanong, ano nga ba ang etikal sa pagsasalin? Mayroon bang mabuti at buktot na pagsasalin?

Nagsisimula ang halagahan ng pang-among moralidad sa amo. Ang malakas ay mabuti, ayon sa amo, dahil malakas ang amo. Ang pang-among moralidad sa pagsasalin, nagsisimula hindi sa isinasalin, kundi sa salin. Ang pagkamapaglaro ay mabuti, sa salin, dahil mapaglaro ang salin. Ang pangamong pagsasalin ay aktibo. Ang pang-aliping pagsasalin ay reaktibo. Bukod sa obsesyon sa pagiging tapat, na binanggit ko na sa itaas, nakaangkla ang identidad ng pang-aliping salin sa pagiging hindi katulad, kontra o anti, sa ibang salin. Ito marahil ang kaso ni Walter Kaufmann, ang pangunahing tagasalin ni Nietzsche sa Ingles pagkatapos ng Ikalawang Digmaang Pandaigdig. Puro siya kritisismo sa mga naunang nagsalin sa kanya. Sa "A Note on This Edition” ng koleksyong Basic Writings of Nietzsche, sinabi ni Kaufmann na "I have used Clifton Fadiman's early translation of The Birth of Tragedy, done when he was a graduate student, as a basis for some parts of my new version. But even where I did not start from scatch, I have compared every sentence with the original, and my revisions are so extensive that the new version is probably more different from his than most Nietzsche translations-including Fadiman's-are from those that preceded them" (xvii). Sa kanyang "Translator's Preface" sa Lampas sa Mabuti at Buktot, tuloy lang ang kritisismo ni Kaufmann, "In preparing the present edition, I hoped at first that I might merely revise her version [i.e. ang salin sa Ingles ni Helen Zimmern], modernizing her somewhat Victorian prose and correcting mistakes; but I soon gave up. The mistakes were too numerous, and in Nietzsche's case nuances are so important that it would be difficult to say at what point an infelicitous rendering becomes downright wrong” (186). Mas malala ang jajment ni Kaufmann sa salin ni Marianne Cowan. Hindi rin daw kasi ito pilosopo tulad ni Zimmern, at bagaman madaling basahin ang bersyon nito, "the merits are somewhat offset by errors of understanding" (186). Tadtad ng talababa ang mga salin ni Kaufmann, at kahit doon ay hindi tumitigil ang kanyang polemiko sa mga naunang nagsalin kay Nietzsche. Halimbawa, sa kanyang salin ng Genealogy, sa ika-11 ng unang sanaysay, sinabi niyang "Francis Golffing, in his free translation of the Genealogy, deletes the blond 
beast three times out of four..." 477). Kailangan nating ilagay sa konteksto si Kaufmann, na isinalin si Nietzsche sa panahong pagkatapos angkupin ang pilosopo ng mga Nazi. Naaawa siya kay Nietzsche. Ito ang kanyang sinabi sa kanyang "Editor's Preface" sa kanyang salin ng Ito ang Salita ni Zarathustra: "Nietzsche's fate in the English-speaking world has been rather unkind, in spite of, or perhaps even in some measure because of, the ebullient enthusiasm of some of the early English and American Nietzscheans. ... And when we look back today, one of the main reasons must be sought in the inadequacies of some of the early translation, particularly of Zarathustra" (Nietzche 107). Bukod sa pag-intindi sa kanyang konteksto, kailangan ding aminin dito, nakakatawa ang mga hirit ni Kaufmann laban sa kanyang mga tinutuligsang karibal (tingnan ang 109-110). Pinakanakakatawa ang hirit niya laban kay R.J. Hollingdale, na kanyang nakakolaboreyt sa pagsasalin ng Genealogy, sa annotated bibliography ng kanyang Nietzsche: Philosopher, Psychologist, Antichrist: "R.J. Hollingdale has translated four works that Kaufmann had done before, and one may wonder why he did not rather attempt some of the works not rendered into English since before World War I” (Kaufmann 494). Malagkit ang hinanakit sa kanyang komento sa Selected Letters of Nietzsche: "when I read the manuscript for the publisher I persuaded Middleton [ang nagsalin ng mga liham] to include a few especially important letters.... I also corrected a great many errors and pointed out that I had only spotchecked the manuscript and that it needed a thorough going over. Unfortunately, nothing was done about that, nor was my help acknowledged in any way" (494). Hindi ito pagtatanggol sa pagiging hindi propesyunal ng maraming tao, o pagmamaliit sa kontribusyon ni Kaufmann sa pag-aaral kay Nietzsche sa Estados Unidos, o kahit sa aking pag-aaral mismo kay Nietzsche. Muli, ito lamang ay pagtatanong, "Bakit ganitong akto, imbes na ganito?" at "Anong uri ng buhay ang pinapahalagahan ng pagpapahalagang ito?” Ang pinapahalagahan ni Kaufmann ay ang pagiging propesyunal na iskolar at masinsin na tagasalin. Tila ba mas tapat pa siya kay Nietzche kaysa ibang relihiyoso sa Bibliya! 


\section{Kasaysayan versus Genealogy}

Sa historiograpiya ni Zeus Salazar, inilarawan niya ang apat na maaaring maging pananaw ng isang nagsusulat ng kasaysayan. Kunwari, sakupin ng Filipinas ang Tsina, at pagkatapos ay mga Filipino ang sumulat ng kasaysayan nila, na ang kausap ay mga Tsino, "kayong mga Tsino ay tamad...", ito ang pangkayong pananaw. Pag naman Tsino ang naglarawan sa mga Filipino sa kanilang mga kapwa Tsino, "silang mga Filipino ay mabangis na mandirigma...”, ito ang pangsilang pananaw. Pangtayong pananaw pag mga Tsino mismo ang nag-usap-usap tungkol sa kasaysayan ng Tsina. "Tayong mga Tsino...” At pag ang mga Tsino ang nagsulat tungkol sa kanilang sarili, pero ang kausap ay mga Filipino, ang tawag dito, pangkaming pananaw.

Malaking bahagi ng Pook at Paninindigan ni Ramon Guillermo ay nakadevowt sa pagtatanggol sa Marxismo laban sa akusasyon ni Salazar, na ito ay diskursong "pangkami" ang pananaw. Reaktibo raw ito, sabi ni Salazar. Kung susundin natin ang terminolohiya ni Nietzsche, ipinoposisyon ng pangkaming pananaw ang Filipinasbilang produkto ng tagalabas. "Kami rin ay..." o di kaya'y "Kami naman ay...” ang palagiang lalabas na pahayag. Paliwanag ni Guillermo, tinutuligsa ni Salazar ang mga makabayan at anti-imperyalistang historyador "dahil sa kanilang pagbibigay-diin sa kapangyarihan 'dayuhan' sa ekonomiya at pulitika ay naipapaliwanag lamang ang mga kaganapang pangkasaysayan batay sa mga panlabas na mga salik at hindi sa pamamagitan ng mga panloob na batayan" (55). Isama na rin natin ang mga kritiko. Tingnan, halimbawa, ang depinisyon ni Bienvenido Lumbera sa "pambansang kultura": "the dynamic aggregate of ideas, traditions, and institutions as these have been concretized by their struggle against colonial rule and neocolonial control" (156, akin ang diin). Mas pang-amo siguro ang pantayong pananaw. "Tayong mga Filipino ay..."

Mas lalo pang lumapot ang isyu, dahil isinangkot ni Salazar ang usapin ng wika. Para sa kanya, pangkaming pananaw ang mga nasyonalistang akdang nakasulat sa Ingles. Ipinagtanggol naman ni Guillermo ang mga manunulat tulad ni Renato Constantino at Jose Ma. Sison, at sinabing sinasalungat ni Salazar ang sarili dahil siya mismo (si Salazar, hindi si Guillermo), ay may 
gawang nakasulat sa banyagang wika. Maaari maging reaktibo, sabi ni Guillermo, sa wikang Filipino, at makabayan sa wikang Ingles.

Ginigiit ni Guillermo na, sa pagpapakahulugan ni Salazar, halos wala nang makakatakas sa kritisismo ng pagiging reaktibo. Sumipi siya ng tatlong Aleman para bigyan ng bigat ang pagtatanggol laban kay Salazar: si Simmel, si Scheler, at si Nietzsche. Para kasi kay Salazar, ang reaktibong kamalayan ang nagpapatatag o lumilikha sa kondisyong mapang-alipin. Pero para kina Nietzsche at Scheler, paliwang ni Guillermo, "ang hindi matinag na katatagan ng mga umiiral na obhektibong kondisyon ng pang-aalipin ang siyang masasabing batayang pinagtutubuan ng diwa ng Ressentiment" (58). Subukan nating harapin ang hamon ni Salazar. Maaari bang magtatag ng bayan nang walang reaktibong elemento, walang pagtukoy sa banyaga? Mito ang kakailanganin dito, hindi lang kasaysayan. Hihingi ako ng tulong mula sa isang kwentista. Masdan ang unang talata ng La India ni Rosario Cruz-Lucero, na nagsisilbing "Henesis" nitong mga konektado niyang maikling kwento (ang kanyang "Bibliya”): "Noong unang panahon, limang bituin ang nahulog sa isang lawa sa tabi ng bayan. O baka mga bulaklak sila, hindi ko alam. Wala akong masyadong alam tungkol sa bituin. Mas kaunti lalo tungkol sa bulaklak. Pero dahil siguro sa pinanggalingan ko, pwede kong sabihing mga sampaguita sila [jasmine para sa mga walang pakialam tungkol sa maliit na pagkakaiba]. At dahil ignorante rin ako tungkol sa swan, sasabihin ko ritong mga swan ang mga bituing ito, kahit wala namang swan sa kagubatan natin. Pero, kung may swan sa kagubatan natin, pwedeng naging swan ang mga bituin na ito. Pinili nila ang bayang ito, dahil dito, maaari nilang ilaan ang anumang panahong ibinigay sa kanila ng Panginoon ng Panahon (na siya ring Diyos ng Araw) para maligo sa lawa. Hindi ko alam kung paano sila naligo kasi bawat isa may sariling paraan ng pagligo" (1; akin ang salin).

Ang naging tuntungan ni Lucero ay mga salita. Nalilipat niya ang tereyn ng tunggalian tungo sa pagalingan ng kwento. Sa ganitong paraan nakukwestyon ni Lucero ang pundasyon ng mga diskursong kanyang natutuligsa. Gayundin, nabibigyan siya ng kalasag nitong kanyang katapatan. Samantalang nagpapanggap ang iba na may mahigpit na pagkahawak sa realidad, inaamin ni Lucero ang hubad na kapangyarihan ng loob na nagtutulak at nagpupum- 
ilit para ipanganak ang realidad na inilalarawan/nililikha. Ang pundasyon ng bawat bayan ay kwento (34-35). Okey na sana. Kaso, na siya namang punto ni Guillermo, may papasok na tagalabas. Ito ang ahas sa paraiso ni Lucero: "May dumaang mangangaso (maaaring Prinsipe ng Liwanag, o ng Kadiliman, meron bang pagkakaiba?) at binosohan sila sa lawa. Masaya siya para sa kanila, ang ibig sabihin, nainggit siya. At dito uusbong ang hidwaan, pero dahil kani-kaniya silang paraan ng pagligo, sasanga ng lima ang banghay" (1; akin ang salin). Namimintis ni Salazar ang alam ng bawat nagtatangkang magsulat ng kwento, dula, o nobela: para tumakbo ang banghay, kailangan ng hidwaan. Laging may nasa labas na siyang magtitriger ng aksyon, na reaksyon. Lahat ng aksyon ay reaksyon. Pwedeng sabihin ni Salazar na hindi kwento ang kanyang isinusulat kundi kasaysayan. Pero narito ang kanyang banghay: noong unang panahon, puro mga pangkaming pananaw ang ginagamit ng mga historyador, hanggang sa isang araw, isang magiting na paham, nagngangalang Zeus, ang tumindig upang ipagtanggol ang Pilipinas mula sa kamalayang pang-alipin. Magugustuhan siguro niya ang inulat ni Lucero di-umano ay mito ng mga Manobo: pitong beses daw lumibot ng mundo ang prinsipeng si Baybayan, inaawit ang kasaysayan ng kanyang bayan (9). Ito ang dahilan kung bakit pare-pareho ang mga kwento, mula dakilang baha hanggang prinsipeng nabilanggo. Ang natatagpuan natin lagi sa iba ay ang ating mga sarili. Pinaglaruan pa lalo ito ni Lucero sa ibang bahagi ng kanyang La India. May paparating kasing peste/salot, at bilang oportunista, kinumisyon ng tauhang si Padre Duertas ang kanyang indiong eskultor na gumawa ng estatwa ni San Roque. Kaso nga lang, sa pagmalas sa larawan ng kanyang gagawan ng eskultura, nabasa ng tagaukit na si Amador ang vida ng santo. Nagustuhan niya ito, at ibinahagi sa asawa. "Hinaharap siya ng yakap-yakap niyang si Susana, na bumulong nang sabik, "Pero iyon ang ating prinsipe, Amador!” At sinimulan ni Susana ang malambing na pagkanta ng kwento ng kanilang Prinsipe Labaw Donggon, kwentong inawit sa kanya ng kanyang lola habang katabi siya nito sa habihan, ginagabayan ang mga kamay ng batang babae" (13; akin ang salin). Ang nangyari: mukhang indio ang ginawang eskultura ni Amador. Dinakip siya ng guardia civil at iniligtas ng mga buyung. Dumating ang salot at naganamatay ang mga bata. Nang 
magkasakit ang anak ng gobernador, dinala niya ito sa indiong San Roque ni Amador. Gumaling ang bata.

Tila ba nalalayo na tayo sa kasaysayan. Pero mas malapit naman ang pilosopiya sa pagkukwento, kumpara sa historya. Ang kasaysayan, nga naman, ay kailangang nakasandig sa datos. Ang kwento, sa salita. At ang pilosopiya? Bilang pilosopo, hindi hihindi si Nietzsche sa mga kwento. Ang Ito ang Salita ni Zarathustra ay hindi streytforward na sanaysay, kundi mala-Bibliyang deklarasyon at aral. Ang pamosong "kamatayan ng Diyos" at "walang hanggang pag-uulit" ay sa anyo ng kwento unang prinsenta sa Ang Maligayang Agham. At ang Genealogy? Puno ito ng kwento. Meron pa ngang diyalogo, mistulang dula. Nabigyang-diin na ang halaga ng estilo kay Nietzsche. Ituloy-tuloy na natin at basahin siya bilang literatura, alinsunod sa mga iskolar tungkol kay Nietzsche.

Istilo ang binigyang-empasis ng maraming espesyalista sa paksang si Nietzsche. Halimbawa'y si Alexander Nehamas sa kanyang Nietzsche: Life as Literature. Ayon sa kanya, nagsulat si Nietzsche sa maraming genre (tula, aporismo, sanaysay, kwento [Ito ang Salita ni Zarathustra], autobiography) dahil "he wants his readers to accept his views, his judgments and his values as much as he wants them to know that there are essentially his views, his judgments, and his values" (35; sa kanya ang emphasis). Konektado ito sa punto ni Nehamas na ang paglikha sa sarili ang sentrong problema ni Nietzsche. Syempre, kung lilikha ng sarili ang isang tao, kailangan sarili nyang gawa ito (hindi mo sarili ang gawa ng iba). Ang empasis ni Nietzsche sa presentasyon, sa istilo, ay ang empasis nya sa kung ano ang kanyang pinepresenta, sa kanyang sinasabi, na dapat gamitin ang sariling isip, at huwag magpaalipin sa mga ideya mula sa iba.

Isa pang katangiang nakita ni Nehamas kay Nietzsche ay ang sobra-sobrang gamit ng pilosopo sa hyperbole. Ayon sa kanya: "It is, for example, one thing to claim that Greek tragedy came to its end through the dramas of Euripides, that the genre was exhausted by them, that tragedy had nowhere to go after Euripides wrote. It is quite another to write, and to mean, as Nietzsche does, that "Greek tragedy... died by suicide... tragically," that Euripides actu- 
ally killed it, and that he used and was used by "aesthetic Socratism as the murderous principle" (22).

Pangatlong katangian at, kung aalalahanin ang pamagat ng kanyang libro, ginawang sentral na problema ni Nehamas ang paggamit ni Nietzsche sa mga talinghagang pampanitikan. Ito'y dahil estetiko ang pananaw ni Nietzsche sa buhay, proyektong nililikha. Uusigin ko ang pagtinging ito ni Nehamas sa ibabang bahagi, sa ikatlong kritisismo ko kay David, tungkol sa walang-hanggang pagbabalik/pag-uulit-ulit.

Istilo rin ang binigyang empasis ni Bernd Magnus, Stanley Stewart, at Jean-Pierre Mileur. Tinukoy nila ang limang estratehiya ni Nietzsche sa kanyang mga sinulat. Ang una'y tugma sa pananaw ni Nehamas, ang paggamit ni Nietzsche sa hyperbole. Ang natitirang apat: "Second, there is the feature of undecidability which inhabits almost all of the writings as a sort of spectral presence. Third, Nietzsche's writings resist paraphrase and they do so in a special way. Fourth, most of his published writings virtually embody the theses for which they argue, they represent, incarnate their thought, they are instances of their own kind, toke of their own type; they are self-referring simulacra... Fifth, a defining feature of Nietzsche's central philosophical gestures is that they are self-consuming concepts" (16).

Patuloy pa nina Magnus, ang estilo ni Nietzsche'y hindi binibigyang empasis ng ibang manunulat. Halimbawa na lang ang Ito ang Salita ni Zarathustra. Ito ay kwento tungkol sa tauhang si Zarathustra, pero tinuturing ng maraming mambabasa na lahat ng sinabi ng tauhang si Zarathustra ay opinyon din ni Nietzsche. At hindi lang 'yon, pati ang mga sinasabi ng ibang tauhan sa kwentong ito'y tinuturing na pananaw ni Nietzsche. Halimbawa nina Magnus ang mga katagang, "You are going among women? Do not forget your whip!" Ito ay binanggit ng matandang babaeng kausap ni Zarathustra, ngunit ginagamit ng ibang manunulat bilang patunay ng sexismo ni Nietzsche.

Mga argumentong ad hominem naman ang istilong nakita ni Robert Solomon sa mga sulatin ni Nietzsche. Sa kanyang "Nietzsche ad hominem: Perspectivism, personality and ressentiment" nilarawan ni Solomon si Nietzsche sa ganitong paraan: "He wondered what made people 'tick,' and he 
rightly suspected that what they thought and said about themselves and their ideals was almost always misleading, mistaken, or just plain fraudulent" (81). Ang isyu para kay Nietzsche, para kay Solomon, ay hindi ang kasagutan sa mga pilosopikal na tanong, kundi: anong uri ng tao ang mag-iisip ng ganitong uri ng mga tanong, at anong klase ang sasagot ng ganito, at anong uri ang sasagot ng ganoon. Ito ang pagiging sikolohista ni Nietzsche.

Kung gayon ay may tatlong tusok ang Nietzscheng ito, bumubuo ng tatsulok. Pilosopiya, dahil nais nating gamitin ang kanyang mga konsepto. Panitikan, dahil magpopokus tayo sa kanyang istilo. At pagsasalin, na siyang tusok sa tuktok, ang siyang kumakain sa dalawa pa.

\section{Pagsasalin bilang Malikhaing Pagsulat}

Sa malikhaing pagsulat, may mga alituntunin at standard na di umano'y unibersal at siyang nagtatakda kung ano ang maganda at kung ano ang pangit. Kahit ang mga mas interesado sa nilalaman ng akda, iyong mga ang pinupuri ay mga gawang may kamalayang panlipunan, sumasangguni pa rin sa mga standard na ito ng kagandahan. Si Kris Montañez, halimbawa, na nagmumula ang kritisismo ng nobelang Hulagpos ni Mano de Verdades Posadas sa paniniwalang may kaisahan dapat ang anyo at nilalaman, dumudulog pa rin sa mga alituntunin ng porma. Buod nga ni Caroline Hau: "... the characters in Hulagpos appear to be mere 'outlines' of life, that these characters seem to derive from propaganda textbooks rather than from their delineation as individual fleshed-out characters.... The sheer number of characters whose narratives need development results in perfunctory interiorization and contextualization, leaving a number of details totally unaccounted for" (265). O: manong NPA ka, kailangan pa rin nang akmang karakterisasyon!

Bagaman sa panlabas na magkaiba itong dalawang pananaw sa panitikan, “sining para kanino?" versus "sining para sa sining," maaamoy nating may iisa silang pundasyon. Tila ba may Platonikong Anyo ng sining na dapat tugmaan ng gawa para ito maging maganda o epektibo. Nauuna ang standard ng krititismo, at sinusubukan itong habulin o tapatan ng manunulat. Mayroong dalawang reaksyong maaaring gawin dito. Una, sundin ang mga alituntunin. Sugal pa rin ito, kasi kahit subukan mong akmaan ang standard, pwede ka 
pa ring mabigo. Pangalawa, pwede kang magrebelde laban sa establishment. Mas mapanganib ito. Una, pinaparusahan ang mga anti-social. Pangalawa, baka akala mo lang may bago kang nalikha, pero iyon pala pareho lang ng mga lumang sumunod sa standard. Pangatlo, walang papansin sa iyo. Pangapat, ampangit ng ginawa mo. Pero, paano naging pangit kung wala namang sinusubukang akmaang alituntunin? Kung ganito ang pangangatwiran, nga lang, pwedeng sabihing paano naging maganda kung wala namang standard na magsasabing maganda ang akda?

Nakikipagsayawan tuloy ang manunulat sa mga alituntunin. Sa isang banda, kailangan niyang sundin ang mga ito. Sa ibang banda, kailangan niyang pakinggan ang sarili, na ito imbes na iyon ang magiging maganda, o epektibo. Ito ang relasyon ng manunulat sa kritisismo. Pero ang krititismong itong kritisismong naghuhusga. Meron pang ibang uri ng krititismo. Ang mahalaga para sa akin ay ang krititismong nagpapaliwanag. Kung, halimbawa, ang kay Montañez, na, kaklaruhin ko, ay pinupuri ang pagkakasulat at sang-ayon sa ideolohiya ng Hulagpos, ay iyong kritisismong nagsasabing may kakulangan ang akda dahil masyadong marami ang mga tauhan at hindi sila naging kapani-paniwala bilang tao, ang kritisismong naghuhusga, sa kritisismong nagpapaliwanag baliktad ang relasyon. Kaya masyadong maraming tauhan sa Hulagpos ay dahil gerilya ang nobelistang nagsulat nito, at iba ang hinihiling na kanyang target audience. Alam na natin kung ano ang nangyayari kung susundin ng manunulat o hindi ang kritisismong nanghuhusga. Maaari niyang masapul o mamintisan ang standard. Ano ang mangyayari kung susundin niya ang kritisismog nagpapaliwanag? Iniwan na niya ang usapin ng ganda o pagkaepektibo-at meron na namang ipapaliwanag ang kritiko. Samantalang nagtatapos ang kritisismong naghuhusga, walang hanggang ang kritisismong nagpapaliwanag. Walang hanggang ang pagpapaliwanag kaya walang hanggang ang pagsusulat.

Ipagpalagay nating tama ito, ano ang kinalaman nito sa pagsasalin? Sa pagsasalin, ang kritisismong naghuhusga ay iyong naglilista ng mga pagkakamali. Mali itong "house" dahil "tahanan" ang orihinal, dapat "home." O di kaya'y "dapat hinati na lang ang pangungusap na ito, dahil ang sapat lang sa Ingles ay masyadong mahaba sa Filipino.” Halos wala nang nagsasayang ng 
oras sa ganitong uri ng kritisismo. Samantala, ang kritisismong nagpapaliwanag, sa pagsasalin, imbes na maglista ng mali, naglalarawan ng mga mutasyon. Halimbawa nga iyong kay Ramon Guillermo, na binasa ang paggamit ni Rizal, sa Guillermo Tell, ng iba't ibang salita para isalin ang "Natur" ni Schiller, sa Wilhelm Tell, sa konteksto ng absens ng konsepto ng "likas na karapatan" sa bokabularyo at veltanshawung Tagalog $(145,185)$. Inilarawan naman ni Guillermo, nang may pagkiling sa pagbasa ni Resil Mojares, ang salin ni Joaquin Tuason ng Ang Bagong Robinson mula sa El Nuevo Robinson ni Tomás de Iriarte, na salin ng Robinson der Jüngere ni Joachim Heinrich Campe, sa konteksto ng paghihirap at pagtitiis sa hirap sa ilalim ng kolonyalismo (41). Naging tuloy itong tratadong moral-relihiyoso.

Parehong aminado sila Iriarte at Tuason sa pagdaragdag, pagwawasto, at pagbabawas sa kanilang mga isinalin (12-13). Pagsasalin pa bang maituturing ang kay Tuason, na nagtanggal ng mga sa tingin niya'y walang silbi sa mga Tagalog at nagdagdag ng mga dasal sa Birheng Maria kung saan wala naman sa orihinal? Pero orihinal ba itong kay Iriarte, na salin lang din naman ng kay Campe? At isa pa, nagiging tapat lang naman si Tuason sa praktika nga ni Iriarte. Ano't ano pa man, makikita rito ang pagkakaiba ng pagsasaling susunod sa kritisismong nanghuhusga sa pagsasaling susunod sa kritisismong nagpapaliwanag. Nagiging walang hanggan ang komentaryo, ang kritisismo. At nagiging walang hanggan ang pagsasalin. Bakit nga naman kakailanganing magsalin pa ulit ng gawa ni Campe o ni Nietzsche o ni Rizal sa Filipino kung nakagawa na si Tuason, Eliserio, Almario ng defenitiv na salin? Sa ipinapanukala kong pagsasalin, paghahaluin ang pagsasabanyaga at pagsasa-Filipino ng mga estruktura ng pangungusap. Mangingitlog nang mangingitlog ng mga korapshon, ng mga salitang shokoy. Hindi matatapos ang pagsasalin, at hindi matatapos ang pagsusulat ng mga salin, ang pagsusulat ng mga kritisismo, at pagsusulat mismo. 


\section{Mga Sanggunian}

Aguilar, Faustino. Nagalunod sa Katihan. Limbagan ng Cultura Filipina, 1911.

Deleuze, Gilles. Nietzsche and Philosophy. Continuum, 1983.

Fraser, Giles. Redeeming Nietzsche. Routledge, 2002.

Guillermo, Ramon. Pook at Paninindigan. UPhilippines P, 2009a.

---. Translation and Revolution. Ateneo de Manila UP, 2009b.

---. Themes of Invention, Help, and Will: Joachim Campe's Robinson der Jüngere in Tagalog and Bahasa Melayu Translations. Southeast Asian Studies 3 (1): 3-47, 2014.

Hau, Caroline. Necessary Fictions. Ateneo de Manila UP, 2000.

Javellana, Rene. "Kasaysayan at mga Suliranin sa Pagsasalin ng mga Tekstong Klasiko."Nasa Salin-Suri: Panimulang Pagmamapa ng mga Larangan ng Pag-aaral ng Pagsasalin sa Filipinas, inedit ni Galileo Zafra,pp. 157-164,University of the Philippines Sentro ng Wikang Filipino, 2009.

Kaufmann, Walter. Nietzsche: Philosopher, Psychologist, Antichrist. PrincetonUP, 1974.

Lucero, Rosario. Ang Bayan sa Labas ng Maynila. Quezon City: Ateneo de Manila UP, 2007.

---. La India. Quezon City: U of the Philippines P, 2012.

Lumbera, Bienvenido. Revaluation. University of Santo Tomas PublishingHouse, 1997.

Magnus, Bernd, Stanley Stewart, at Jean-Pierre Mileur. Nietzsche's Case: Philosophy As/And Literature. Routledge, 1993.

Nehamas, Alexander. Nietzsche: Life as Literature.Harvard UP, 1985.

Nida, Eugene. "Principles of Correspondence."Nasa The Translation Studies Reader, inedit ni Lawrence Venturi, pp. 126-140. Routledge, 2000.

Nietzsche, Friedrich. The Portable Nietzsche. Inedit ni Walter Kaufmann. Penguin, 1959.

---. Basic Writings of Nietzsche. Inedit ni Walter Kaufmann. The Modern Library, 1967.

Schacht, Richard. Introduction. Nietzsche, Genealogy, Morality, inedit ni Richard Schacht,pp. ix-xvi. U of California P, 1994.

Solomon, Robert. "Nietzsche ad hominem: Perspectivism, Personality and Ressentiment Revisited." Nasa The Cambridge Companion to Nietzsche, inedit nina Bernd Magnus at Kathleen Higgins,pp. 180-222. Cambridge UP, 1995.

Solomon, Robert, at Kathleen Higgins. What Nietzsche Really Said. Schocken, 2000. 